\title{
Admission Control for Multiservices Traffic in Hierarchical Mobile IPv6 Networks by Using Fuzzy Inference System
}

\author{
Jung-Shyr Wu, ${ }^{1}$ Shun-Fang Yang, ${ }^{1,2}$ and Chen-Chieh Huang1 \\ ${ }^{1}$ Department of Communication Engineering, National Central University, Chung-Li 32001, Taiwan \\ ${ }^{2}$ Telecommunication Laboratories, ChungHwa Telecom Co., Ltd., Yang-Mei 32601, Taiwan \\ Correspondence should be addressed to Shun-Fang Yang, ysf@cht.com.tw
}

Received 16 March 2011; Accepted 26 September 2011

Academic Editor: Youyun Xu

Copyright (C 2012 Jung-Shyr Wu et al. This is an open access article distributed under the Creative Commons Attribution License, which permits unrestricted use, distribution, and reproduction in any medium, provided the original work is properly cited.

CAC (Call Admission Control) plays a significant role in providing QoS (Quality of Service) in mobile wireless networks. In addition to much research that focuses on modified Mobile IP to get better efficient handover performance, CAC should be introduced to Mobile IP-based network to guarantee the QoS for users. In this paper, we propose a CAC scheme which incorporates multiple traffic types and adjusts the admission threshold dynamically using fuzzy control logic to achieve better usage of resources. The method can provide QoS in Mobile IPv6 networks with few modifications on MAP (Mobility Anchor Point) functionality and slight change in BU (Binding Update) message formats. According to the simulation results, the proposed scheme presents good performance of voice and video traffic at the expenses of poor performance on data traffic. It is evident that these CAC schemes can reduce the probability of the handoff dropping and the cell overload and limit the probability of the new call blocking.

\section{Introduction}

Since the next generation networks will be unified networks based on IP architecture, the design of IP-based mobility management schemes becomes necessary. The IETF MIPv6 (Mobile IPv6) [1] and its extension were proposed for efficient mobility management. HMIPv6 (Hierarchical MIPv6) [2] manages the mobility of an MN (Mobile Node) using both a router located in the MN's home domain and a router located in a domain visited by the MN. Local movements of the MN are hidden from the outside of the visited domain. The HMIPv6 can reduce the amount of signaling and improve the performance of handover latency. Although much research $[3,4]$ focuses on modified Mobile IP to get better efficient performance, there are few research papers to discuss admission control scheme when considering handover and mobility management [5].

CAC (Call Admission Control) has to be revised to deal with the anticipated new composite radio wireless environment [6]. The ratio of the reserved bandwidth is adjusted to make CAC in the Mobile IP networks in [7]. Because the blocking probability, dropping probability and cell utilization are sensitive to the reserved bandwidth, it is challenge in deciding how to adjust this ratio. The utilization of the wire line link between HA (Home Agent) and FA (Foreign Agent) is used to make CAC in $[8,9]$. Because the wireless links are much easier to be the bottle neck than the wire line link, it is not proper to use the utilization of the wire line link alone. Good CAC schemes have to balance the new call blocking and the handoff call blocking in order to provide the desired QoS (Quality of Service) requirements. Since the channel reservation can be adjusted dynamically, mobility-based call admission control schemes can be designed to provide QoS in the wireless networks [10].

The GCP (Guard Channel Policy), proposed in [11], keeps a certain amount of channels to handoff calls only while the rest of the channels can be shared by both new calls and handoff calls. It has been shown in [12] that the GCP can minimize a linear objective function of $\mathrm{Pb}$ (new call blocking probability) and Pd (handoff dropping probability). An enhanced version of guard channel policy, called FGCP (Fractional GCP), has proven to be optimal in minimizing $\mathrm{Pb}$ with a hard constraint on $\mathrm{Pd}$ and minimizing 


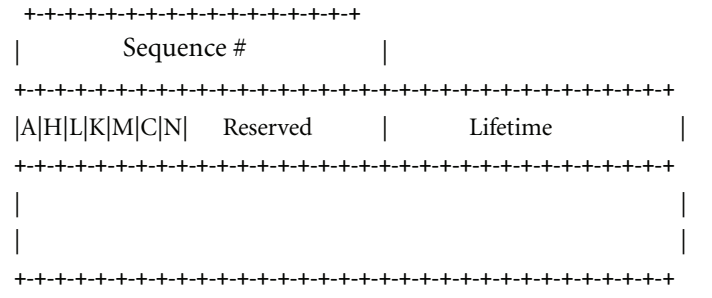

FIgURE 1: Local Binding Update message.

the number of required channels with a hard constraint on both $\mathrm{Pb}$ and Pd. LFGCP (Limited FGCP), a combination of GCP and FGCP, is shown to be more effective than the basic guard channel policy in minimizing $\mathrm{Pb}$ and the number of required channels while holding the constraint on $\mathrm{Pd}$.

In addition to much research that focuses on modified Mobile IP to get better efficient handover performance, CAC should be introduced to Mobile IP-based network to guarantee the QoS for users. The CAC schemes should handle packet-based applications that have highly burst and nonstationary traffic in modern communication networks. In this paper, we propose a CAC scheme which incorporates multiple traffic types and adjusts the admission threshold dynamically using FIS (Fuzzy Inference System) to achieve better usage of resources. The rest of the paper is organized as follows. Section 2 presents our previous work in the area of the HMIPv6-based CAC schemes. The proposed admission control algorithm is described in Section 3. The simulation architecture and result discussion are described in Sections 4 and 5, respectively. Finally, Section 6 concludes this paper.

\section{HMIPv6-Based CAC Schemes}

Some of the mobility-based CAC schemes that require extensive knowledge of the system parameters are not easy to implement; moreover, they sacrifice the scarce radio resources to satisfy the deterministic QoS bounds. The HMIPv6 extension headers can be combined with wellknown CAC schemes, and the impacts of terminal mobility to the network performance are discussed. Our proposed method only requires few modifications on MAP (Mobility Anchor Point) functionality and slight change in BU (Binding Update) message formats.

The guard channel schemes are expanded to combine with the mobility information. A new call or a handoff call is identified from the new registered or updated HMIPv6 BU messages. The movement of MNs can be predicted by observing the variation of MN's position in a fixed period. In Figure 1, a new flag $\mathrm{C}$ is added in HMIPv6's BU message format, and it provides the movement recording of MNs for the calculation of the movement patterns. The $\mathrm{M}$ flag is defined in HMIPv6, and it indicates MAP registration. When an $\mathrm{MN}$ registers with the $\mathrm{MAP}$, the $\mathrm{M}$ and $\mathrm{A}$ flags must be set to distinguish this registration from a $\mathrm{BU}$ being sent to the HA or a CN (Correspondent Node). An optional flag N

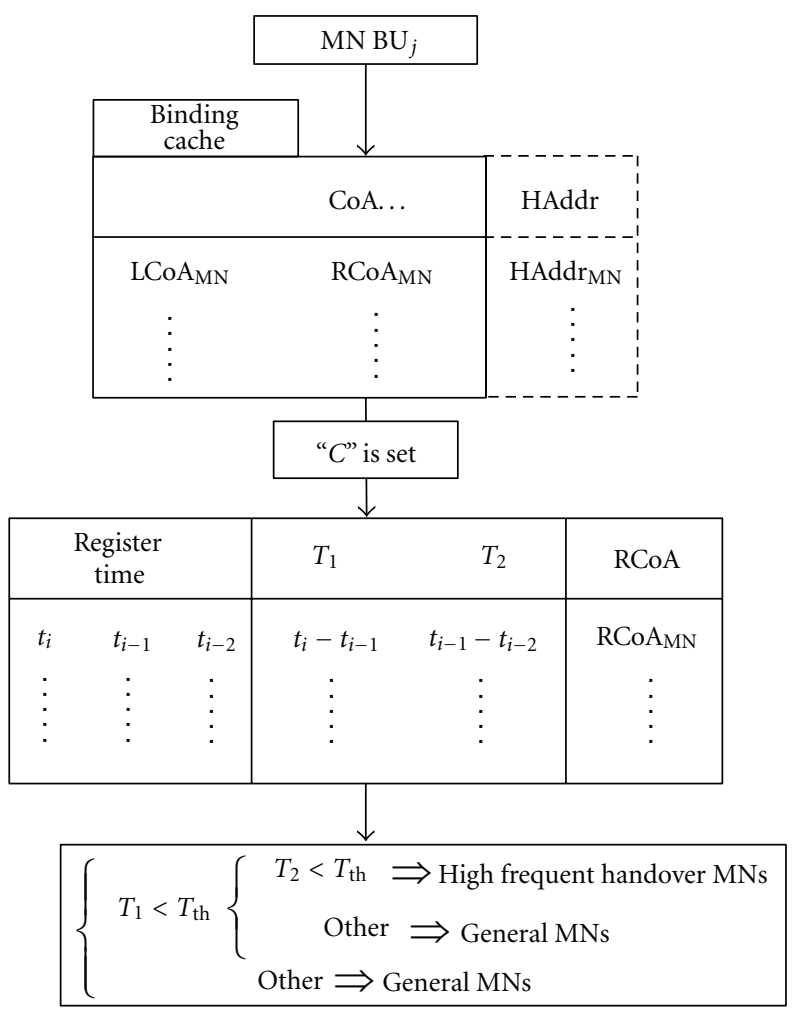

FIgURE 2: The operation procedure of the MAP.

indicates a lower hierarchical MAP registration in the mobile networks. When $\mathrm{C}$ is set, $\mathrm{M}$ and $\mathrm{N}$ should be set in the MAP. The lower hierarchy MAPs will cover many ARs (Access Routers) that located far from the default MAP. When an $\mathrm{MAP}$ receives a $\mathrm{BU}$ from an $\mathrm{MN}$, it will look up its binding cache at first. If there is no record, it will inform other MAPs. If there are no records in all MAPs, the call is identified as a new call. Because the IPv6 supports piggyback, the above procedure can use data messages to reduce the overhead of IPv6 headers.

The operation procedure of the MAP for the BU registration of $\mathrm{MN}$ is shown in Figure 2. When an MAP receives a BU with $\mathrm{C}$ flag set, it records the current registration time $\left(t_{i-2}\right)$, the registration time $\left(t_{i-1}\right)$ for the next times, and the registration time $\left(t_{i}\right)$ for the next two times. The MAP also calculates $T_{1}=t_{i}-t_{i-1}, T_{2}=t_{i-1}-t_{i-2}$. The value of $T_{2}$ and $T_{1}$ represents MN's cell residency time in the current and next handoff cells. We define $T_{\text {th }}$ as the critical threshold residency time. If both $T_{1}$ and $T_{2}$ are smaller than $T_{\text {th }}$, this call is recognized as a high-speed MN. The MAP uses MN' LCoA (on-link Care of Address) to identify which AR serves the MN and calculates the number of high-speed MNs and all MNs. The above procedure can reveal the MN density in the ARs and mobility patterns.

The mobility information can be combined with GCP, FGCP, and LFGCP as three mobility-based CAC schemes. It is evident that a high-speed MN needs much more capacity. When the number of high-speed MN is larger than a certain 


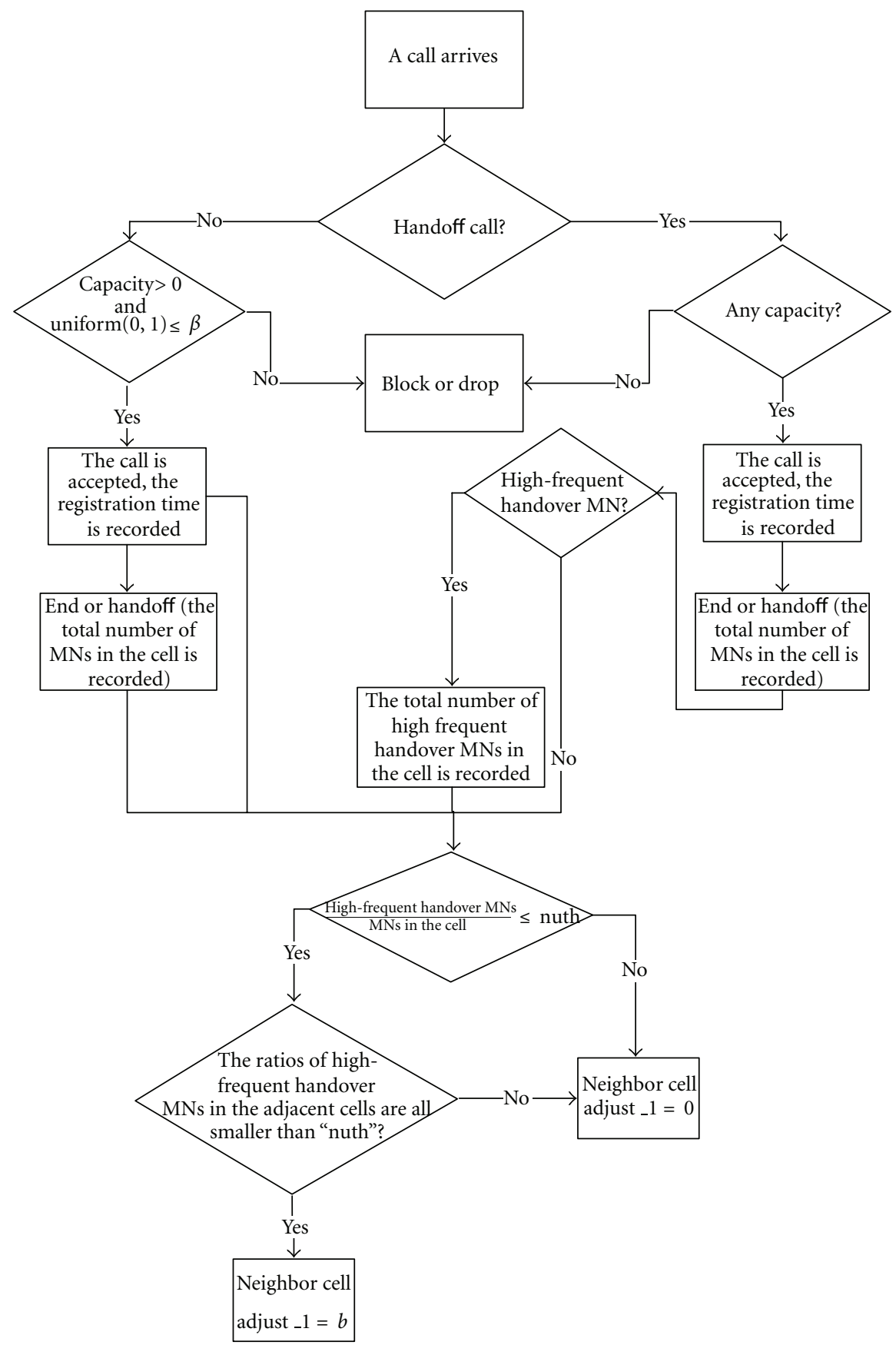

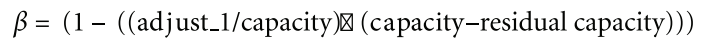

Figure 3: The MBLFGCP CAC scheme.

ratio, the capacity of the cell will be exhausted. The ratio of high-speed MNs (Rhm) is defined to be

The ratio of high-speed MNs

$$
=\frac{\text { (the total number of high-speed MNs in the cell) }}{\text { (the total number of MNs in the cell) }} \text {. }
$$

The parameter "nuth" is defined as the threshold of the ratio of high-speed MNs, the parameter "adjust" as the reserved capacity, and the parameter "adjust_1" as a random adjustable value. The description of MBLFGCP (MobilityBased LFGCP) CAC scheme is shown in Figure 3. The MBLFGCP combines MBGCP (Mobility-Based GCP) and MBLGCP (Mobility-Based LGCP), as same as the LFGCP 


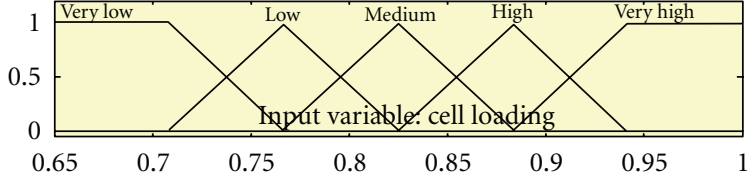

(a)

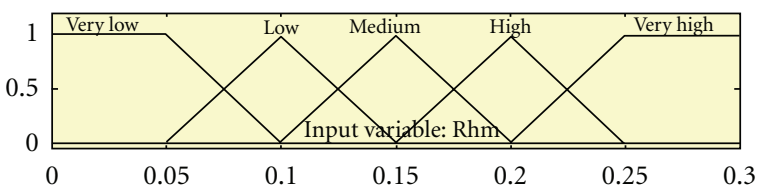

(b)

FIgURE 4: The membership functions of the input fuzzy variables.

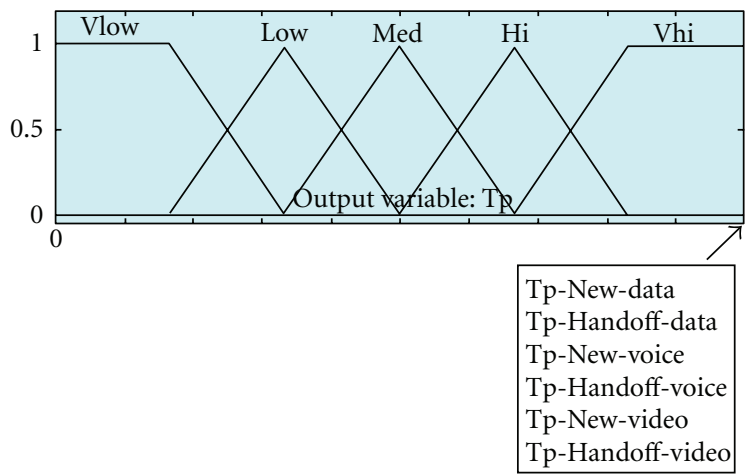

FIGURE 5: The membership functions of the output fuzzy variables.

combines GCP and LGCP. The MBGCP will reject any new call when the parameter "adjust" equals "gc" (the number of free channels). The MBFGCP uses $\beta$ to do admission control of new call. If the ratio of higher-mobility MNs in the adjacent cells is all smaller than "nuth", the parameter "adjust_1" of the center cell will change to $b(0<b<1)$. Then $\beta$ will be set according to the residual capacity of the cell. If it is a new call, the capacity of the service cell should be checked at first. If there is enough capacity, we use probability of $\beta$ to accept a call or reject a call. The MBLFGCP uses $\beta$ to do admission control when the "adjust_1" equals $b$.

The detailed mobility-based CAC schemes and the performance comparison of these CAC schemes have been shown in our previous works $[13,14]$. These CAC schemes only distinguish the priority between new call and handoff call and reserve fixed amount of bandwidth for high-priority traffic. These CAC schemes cannot satisfy the requirements of the present network environment which contains several types of service $[15,16]$. On the other hand, reserving fixed amount of bandwidth will result in worse usage of BS (Base Station) bandwidth [16, 17]. Therefore, we propose a CAC scheme which incorporates multiple traffic types and adjusts the admission threshold dynamically using FIS (Fuzzy Inference System) to achieve better usage of resources.

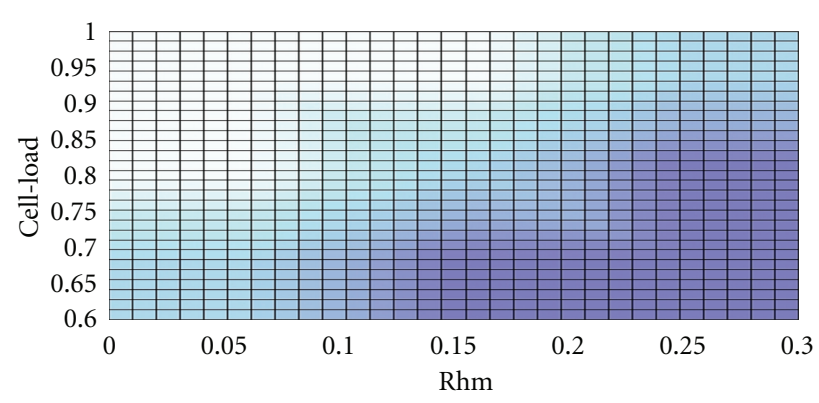

Figure 6: The value of the output variable.

\section{The Proposed Admission Control Algorithm}

Because the packet-based traffic exhibits highly burst and nonstationary properties, the previous CAC schemes cannot handle the drastic changes of traffic. Fuzzy logic is suitable to dynamicly control the thresholds for multiple traffic in the CAC schemes. We assume that there is one BS or several BSs under an AR. For the decision of the threshold values, the cell loading of the candidate BS and the ratio of high-speed nodes in the candidate BS are the input variables of the FIS, and the values of the threshold are the output variables of the FIS.

3.1. Dynamic Fuzzy Control of the Threshold Values for Multiple Traffic. Fuzzy logic, used to explain how to make suitable decision from imprecise and dissimilar information, is a good candidate to deal with the CAC threshold problem because it is able to simplify a large state space of resolution by means of reasonable rules. According to the understanding of the dynamic fuzzy control of the CAC threshold, the objective of the problem is to select the most appropriate threshold values taking into account different inputs.

For the decision of the threshold values, two criteria are used: the cell loading of the candidate BS and the ratio of high-speed nodes in the candidate BS. The cell loading of the candidate BS is defined to be

cell loading

$$
=\frac{\text { (the total bandwidth of all traffic used in the cell) }}{\text { (the total bandwidth capacity in the cell) }} \text {. }
$$

These values of threshold parameter (Tp) could be dynamically changed depending on the system requirements. We define the output parameters as follows.

(A) Tp-New-data $\left(T_{\mathrm{da}}\right)$ : the threshold parameter for admission control of new generated data traffic, where $T_{\mathrm{da}}$ depends on the total number of the voice and video traffic $\left(N_{\mathrm{vo}}, N_{\mathrm{vi}}\right)$.

(B) Tp-New-voice $\left(T_{\text {vo }}\right)$ : the threshold parameter for admission control of new generated voice traffic, where $T_{\mathrm{vo}}$ depends on the total number of the data and video traffic $\left(N_{\mathrm{da}}, N_{\mathrm{vi}}\right)$.

(C) Tp-New-video $\left(T_{\mathrm{vi}}\right)$ : the threshold parameter for admission control of new generated video traffic, 


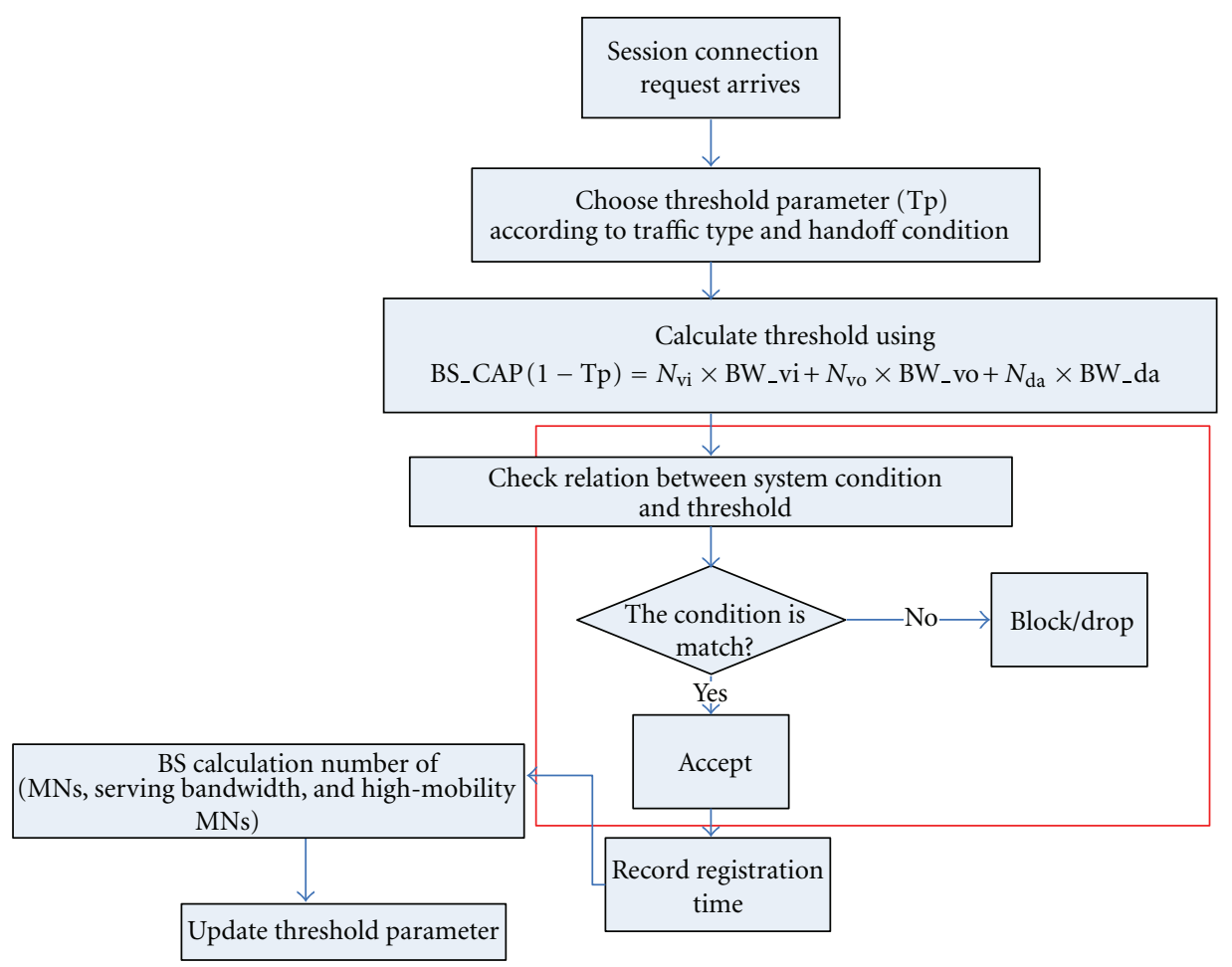

FIgURE 7: The main operation procedure of the proposed algorithm.

where $T_{\mathrm{vi}}$ depends on the total number of the voice and data traffic $\left(N_{\mathrm{vo}}, N_{\mathrm{da}}\right)$.

(D) Tp-Handoff-data $\left(T_{\mathrm{h} \_d a}\right)$ : the threshold parameter for admission control of handoff data traffic, where $T_{\mathrm{h} \_\mathrm{da}}$ depends on the total number of the voice and video traffic $\left(N_{\mathrm{vo}}, N_{\mathrm{vi}}\right)$.

(E) Tp-Handoff-voice $\left(T_{\mathrm{h}_{-} \mathrm{vo}}\right)$ : the threshold parameter for admission control of handoff voice traffic, where $T_{\mathrm{h} \text { vo }}$ depends on the total number of the data and video traffic $\left(N_{\mathrm{da}}, N_{\mathrm{vi}}\right)$.

(F) Tp-Handoff-video $\left(T_{\mathrm{h}_{\text {vvi }}}\right)$ : the threshold parameter for admission control of Handoff video traffic, where $T_{\mathrm{vi}}$ depends on the total number of the voice and data traffic $\left(N_{\mathrm{vo}}, N_{\mathrm{da}}\right)$.

The FIS decision procedures operate in three steps, namely, fuzzification, inference engine, and defuzzification $[18,19]$. The objective of the fuzzification process is to assign, for each input linguistic variable, a value between 0 and 1 corresponding to the degree of membership of the input to a given fuzzy set. The degree of membership values is obtained through membership functions. These input and output variables have five fuzzy sets: "very high" (VHi), "high" (Hi), "medium" (Med), "low", and "very low" (VLow). The membership functions of the input fuzzy variables are shown in Figure 4, and the membership functions of the output fuzzy variables are shown in Figure 5.

For each combination of fuzzy set from the fuzzification step, the inference engine makes use of some predefined fuzzy rules to indicate the suitability of selecting outputs.
TABle 1: The fuzzy logic rules used in the FIS.

\begin{tabular}{llllll}
\hline Rhm Cell-Load & VLow & Low & Med & Hi & VHi \\
\hline VHi & VLow & VLow & VLow & Low & Med \\
Hi & VLow & Low & Low & Med & Hi \\
Med & VLow & Low & Med & Hi & VHi \\
Low & Low & Med & Hi & Hi & VHi \\
VLow & Med & Hi & VHi & VHi & VHi \\
\hline
\end{tabular}

Fuzzy rules were defined as a set of possible scenario utilizing a series of If-Then rules. For example, if the cell loading is "high," and Rhm is "high," the output variable is "medium". The fuzzy logic rules used in the FIS are shown in Table 1. Finally, the defuzzification converts the outputs of the inference engine into a crisp value, that is, a number ranging between 0 and 1 . The other operation methods of the FIS [20] are shown in Table 2. The value of the output fuzzy variable is shown in Figure 6. The relationship between the input and output fuzzy variable has different style for different traffic types. The small value of the output variable, defined as the colored ranges, indicates that the call is easy to be accepted.

3.2. The Main Operation Procedure of the Proposed Admission Control Algorithm. The main operation procedure of the proposed algorithm is shown in Figure 7. The algorithm is described in the following.

When a new or handoff session enters the cell range of a BS, the BS should check the available bandwidth and decides 


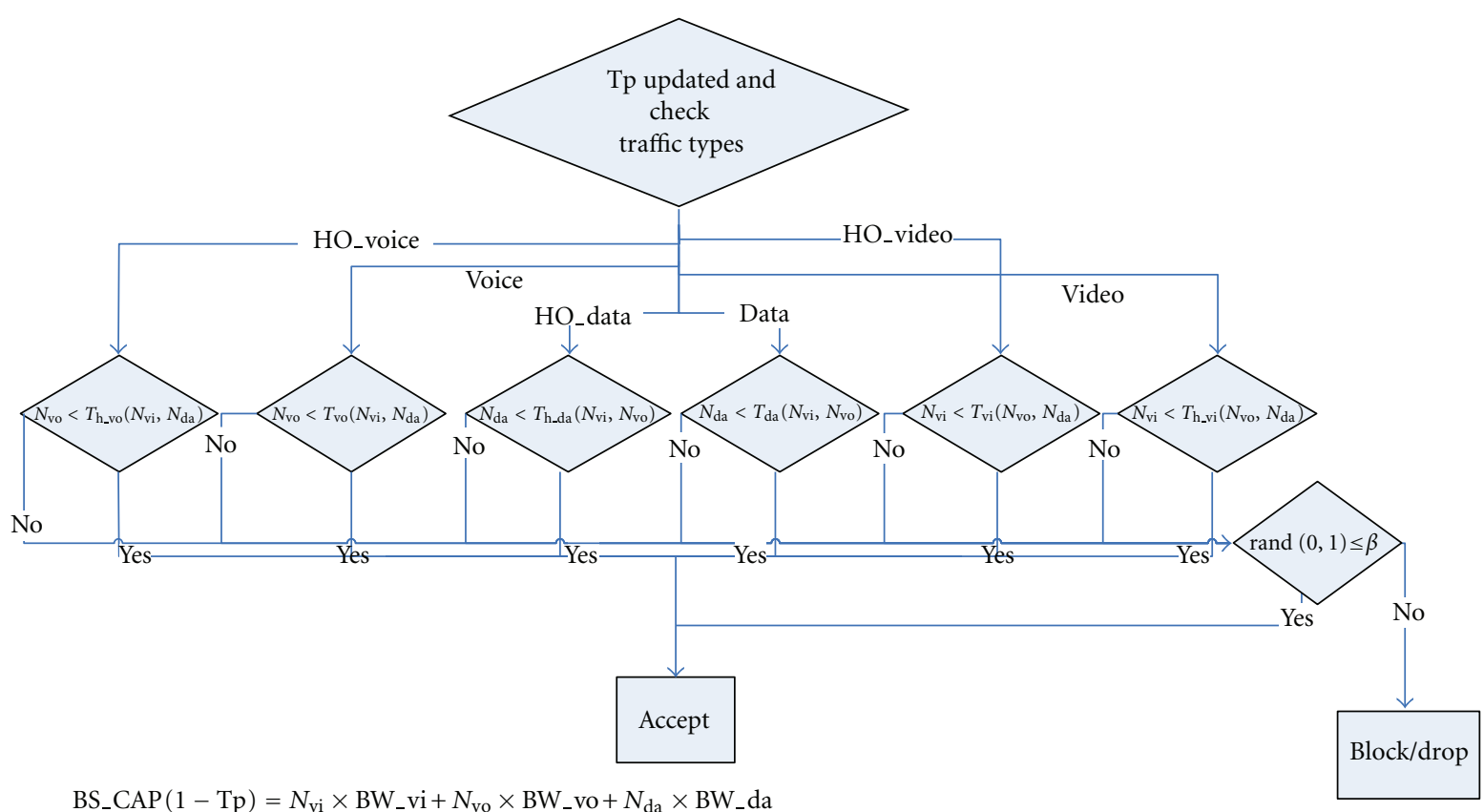

For example: $\mathrm{T}_{\mathrm{vi}}=\frac{\mathrm{BS} \_C A P(1-\mathrm{Tp})-N_{\mathrm{da}} \times \mathrm{BW}_{-} \mathrm{da}-N_{\mathrm{vo}} \times \mathrm{BW}_{-} \mathrm{vo}}{\mathrm{BW} \_\mathrm{vi}}$

FIGURE 8: The scheme used to check the relationship between the system status and the threshold values.

TABLE 2: The operation methods of the FIS.

\begin{tabular}{lll}
\hline Operation & Method & Mathematical formula \\
\hline Intersection & Standard intersection & $t(p, q)=\min (p, q)$ \\
Union & Standard union & $s(p, q)=\max (p, q)$ \\
Implication & Mamdani product implication & $B^{\prime}(y)=\max _{l=1}^{m}\left[\max _{x \in X}\left(A^{\prime}(x) \wedge A_{1}^{l}\left(x_{1}\right) \wedge A_{2}^{l}\left(x_{2}\right) \wedge B(y)\right)\right]$ \\
Aggregation & Max & $y^{*}=\frac{\sum_{i=1}^{k} y_{i} B\left(y_{i}\right)}{\sum_{i=1}^{k} B\left(y_{i}\right)}$ \\
\hline
\end{tabular}

to accept or block this session. If there is available residual bandwidth, the BS should select the suitable threshold parameter according to the traffic type (voice or data or video) and call type (new or handoff). Then the BS uses the selected threshold parameter to do admission control. If the system capacity is enough, this session is to be accepted. Otherwise, the BS uses the probability of $\beta$ to do admission control as MBLFGCP. The other procedures are same as that of the MBLFGCP.

Because the BS should reserve a fraction of bandwidth to accept high-priority sessions, the summation of the bandwidth that occupied by all current traffic sessions should be equal to a part of the total bandwidth. Here, we used the following equation to calculate the value of the selected threshold parameter.

$$
\begin{aligned}
& \text { BS_CAP }(1-\mathrm{Tp}) \\
& \quad=N_{\mathrm{vi}} \times \text { BW_vi }+N_{\text {vo }} \times \text { BW_vo }+N_{\text {da }} \times \text { BW_da, }
\end{aligned}
$$

where $N_{\mathrm{vi}}, N_{\mathrm{vo}}$, and $N_{\mathrm{da}}$ represent the session number of video, voice, and data sessions in a BS, respectively. BW_vi,BW_vo, and BW_da represent the required bandwidth of video, voice, and data sessions in a BS, respectively, and BS_CAP is the total bandwidth of a BS.

The detailed scheme, the region enclosed by the red line in Figure 7, used to compare the system status and the threshold values is shown in Figure 8. For example, the meaning of $N_{\mathrm{vo}}<T_{\mathrm{vo}}\left(N_{\mathrm{vi}}, N_{\mathrm{da}}\right)$ can be explained as follows: if the number of the new voice is smaller than the selected threshold parameter, the session will be accepted, otherwise, the BS uses $\beta$ to do admission control as in MBLFGCP.

\section{Simulation Description}

We use a self-made $\mathrm{C}++$ simulator to compare the performance of the following schemes: no CAC (uncontrolled), LFGCP, MBLFGCP, and the proposed FIS-based CAC algorithm in the Hierarchical Mobile IPv6 networks. 


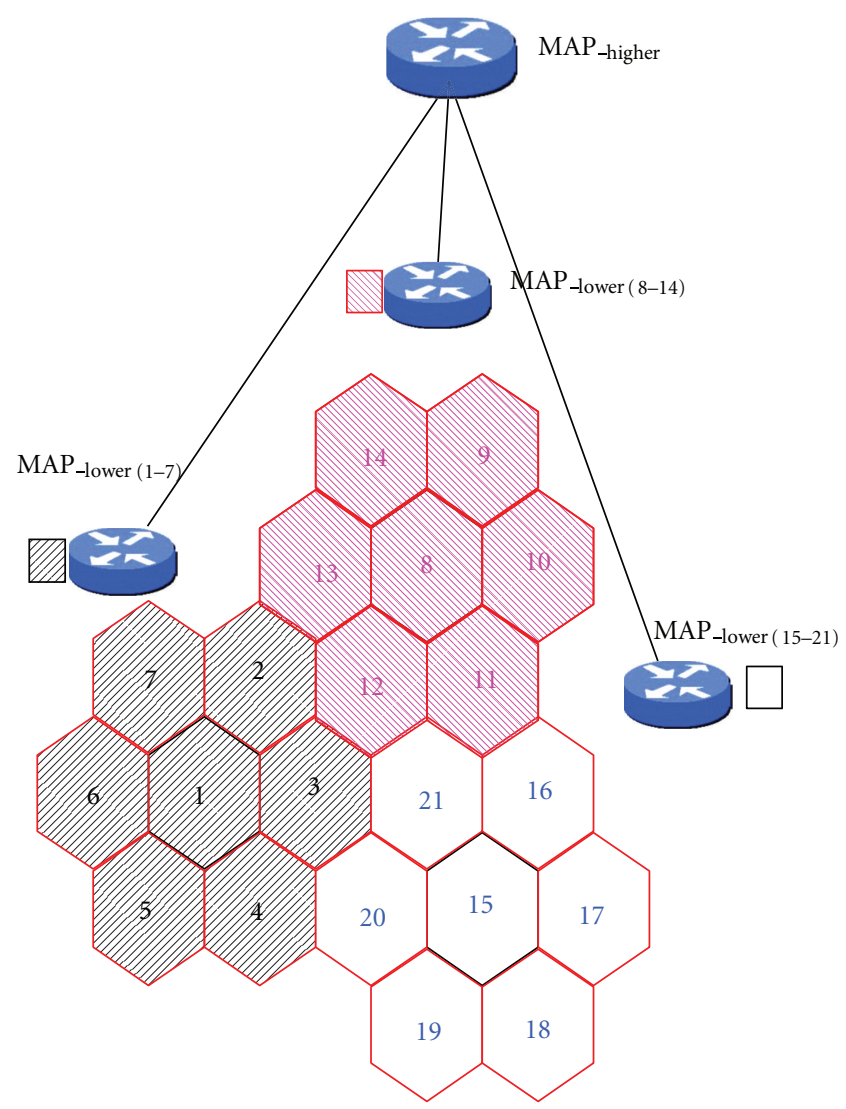

FIGURE 9: The network architecture in the simulation.

The network architecture of the simulation is shown in Figure 9. There are 7 ARs under a MAP (MAP is also an AR), and there are $3 \mathrm{MAP}_{\text {- lower }}$ (Lower Hierarchy MAPs) under a $\mathrm{MAP}_{\text {_higher }}$ (Higher Hierarchy MAP). When an MN registers with the $\mathrm{MAP}_{\text {higher, }}$, the $\mathrm{M}$ flag must be set. When an MN registers with the different $\mathrm{MAP}_{\text {lower }}$, the $\mathrm{N}$ and $\mathrm{M}$ flag must be set. If the movement is in the same MAP_lower, the $\mathrm{N}$ flag must be set. When a BU message with $\mathrm{M}$ and $\mathrm{N}$ flag is sent to $\mathrm{MAP}_{\text {_lower }}$, this BU message will be forwarded to MAP_higher. We assume that AR1-7 are under MAP lower1, AR8-14 are under MAP_lower2, and AR15-21 are under MAP_lower3.

The simulation parameters are shown in Table 3 . Here the video traffic includes only real-time video conference applications. The average session duration of voice, data, and video traffics are 180, 40, and 300 seconds, separately. The percentage of voice, data, and video traffics are $40 \%, 58 \%$, and $2 \%$, separately. We assume that the connection level arrival rate in the AR follows Poisson distribution, and the mean arrival rate varies from 0.4 to 1.1 sessions per second. The average velocities of mobile nodes are from $10 \mathrm{~km} /$ hour to $55 \mathrm{~km} /$ hour with random walk mobility model. To model the movement of the MNs in the system, we assume that the time is slotted, and that the MN can make at most one move during a slot. The movements are assumed to be stochastic and independent from one MN or another. The numerical results take the average of 10 independent simulation runs.
TABLE 3: The simulation parameters.

\begin{tabular}{llll}
\hline & Voice & Data & Video \\
\hline Duration & $180 \mathrm{~s}$ & $40 \mathrm{~s}$ & $300 \mathrm{~s}$ \\
Data rate & $25 \mathrm{kbps}$ & $75 \mathrm{kbps}$ & $400 \mathrm{kbps}$ \\
Percentage & $40 \%$ & $58 \%$ & $2 \%$ \\
Velocity & \multicolumn{3}{c}{$10 \mathrm{~km} / \mathrm{hr} \sim 55 \mathrm{~km} / \mathrm{hr}$ (random walk) } \\
Arrival rate & $0.4 \sim 1.1 \mathrm{sessions} / \mathrm{second}$, Poisson distribution \\
Cell capacity & \multicolumn{3}{c}{$10 \mathrm{Mbps}$} \\
Cell Radius & & $250 \mathrm{~m}$ \\
\hline
\end{tabular}

TABLE 4: The maximum values of the threshold parameters and the range of $\beta$.

\begin{tabular}{lcccc}
\hline Maximum value of Tp & Set-1 & Set-2 & Set-3 & Set-4 \\
\hline Tp-New-data & 0.12 & 0.139 & 0.139 & 0.15 \\
Tp-Handoff-data & 0.085 & 0.115 & 0.115 & 0.102 \\
Tp-New-voice & 0.086 & 0.06 & 0.06 & 0.072 \\
Tp-Handoff-voice & 0 & 0 & 0 & 0 \\
Tp-New-Video & 0.102 & 0.108 & 0.108 & 0.108 \\
Tp-Handoff-video & 0 & 0 & 0 & 0 \\
Range of $\beta$ & $0.17 \sim 0.8$ & $0.25 \sim 0.65$ & $0.3 \sim 0.75$ & $0.25 \sim 0.65$ \\
\hline
\end{tabular}

Three performance indicators are defined in the following:

$$
\begin{aligned}
\mathrm{Pd} & =\frac{\text { handoff dropping sessions }}{\text { all sessions in an AR }}, \\
\mathrm{Pb} & =\frac{\text { new blocking sessions }}{\text { all sessions in an } \mathrm{AR}}, \\
\mathrm{Pb} \& \mathrm{~d} & =\frac{\text { dropping or blocking sessions }}{\text { all sessions in an AR }} .
\end{aligned}
$$

\section{Performance Evaluation}

We use four different fuzzy sets for the performance comparison. The maximum values of the threshold parameters are shown in Table 4 . The maximum values of the threshold parameters are mapped to "very high" (VHi). Because the voice and video handoff traffic have the first priority, TpHandoff-voice and Tp-Handoff-video are both equal to zero.

The LFGCP and the MBLGCP use the $\beta$ value to identify the acceptance probability of a new call or a handoff call. The value curves $\beta$ with respect to the occupied capacity are shown in Figure 10. We set the parameter adjust_1 to be 0.3 and the reserved bandwidth (guard channel) to be $400 \mathrm{kbps}$ ( $4 \%$ of BS_CAP). For example, the acceptable probability of a new call or a handoff call is 1 when the occupied capacity is smaller than the reserved bandwidth and the acceptable probability of a new call or a handoff call is decreased to 0.7 as shown in Figure 10(a).

The comparison of blocking probability of all traffic types is shown in Figure 11, and the comparison of dropping probability of all traffic types is shown in Figure 12. We have compared no CAC (uncontrolled), LFGCP, MBLFGCP, and the proposed FIS-based CAC schemes in the Hierarchical 


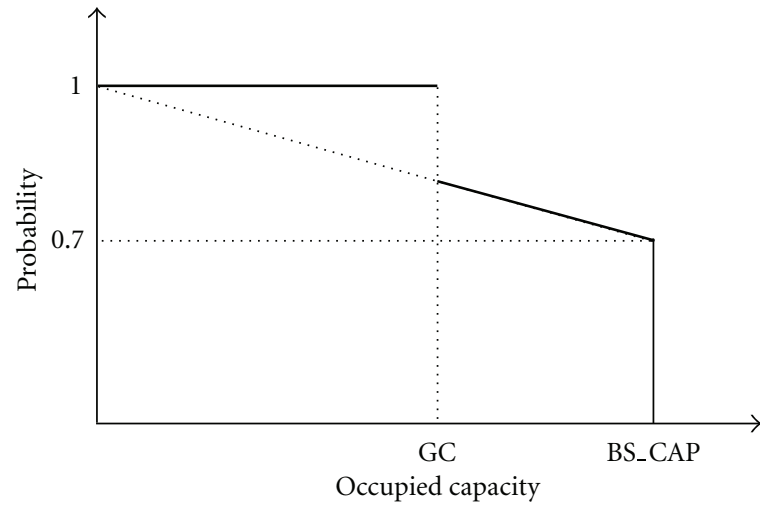

(a) LFGCP, MBLFGCP

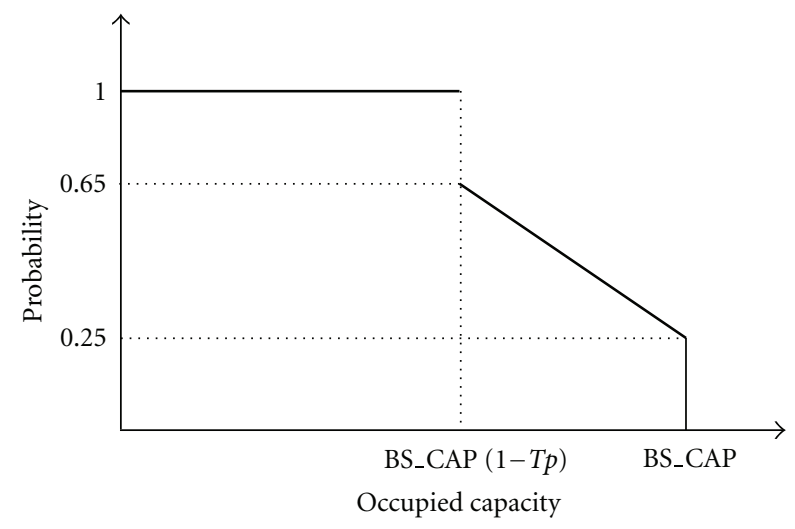

(c) Fuzzy set-2, set-4

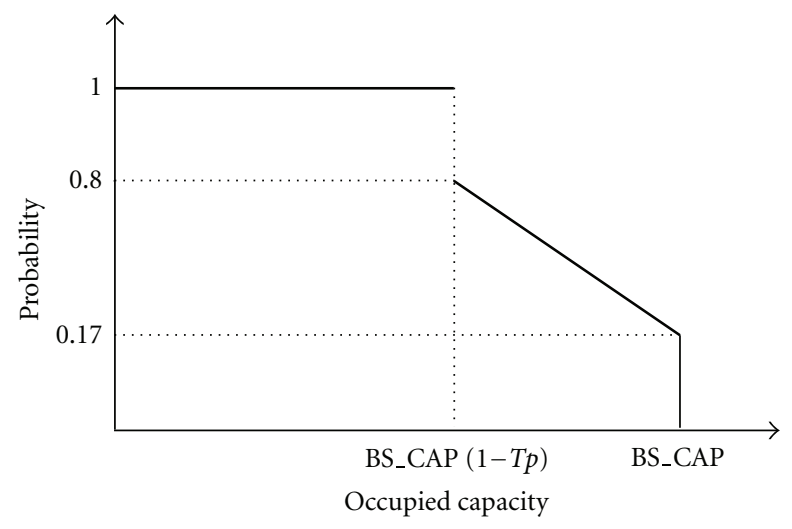

(b) Fuzzy set-1

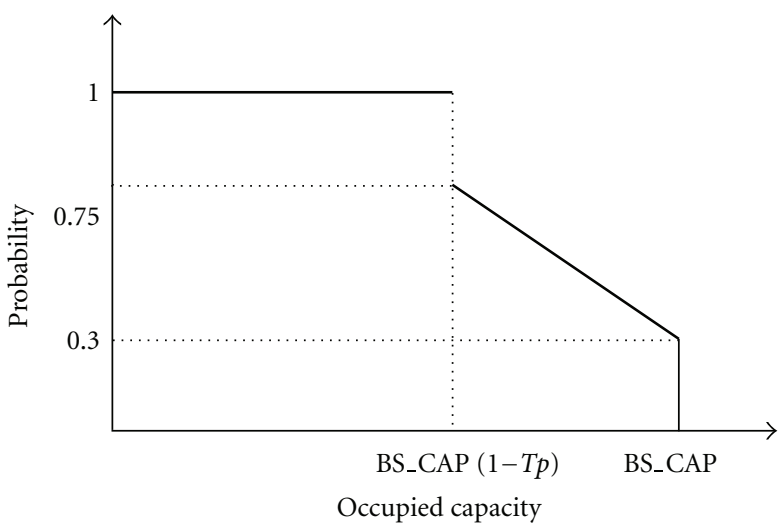

(d) Fuzzy set-3

FIGURE 10: The value curve of $\beta$ probability versus occupied capacity.

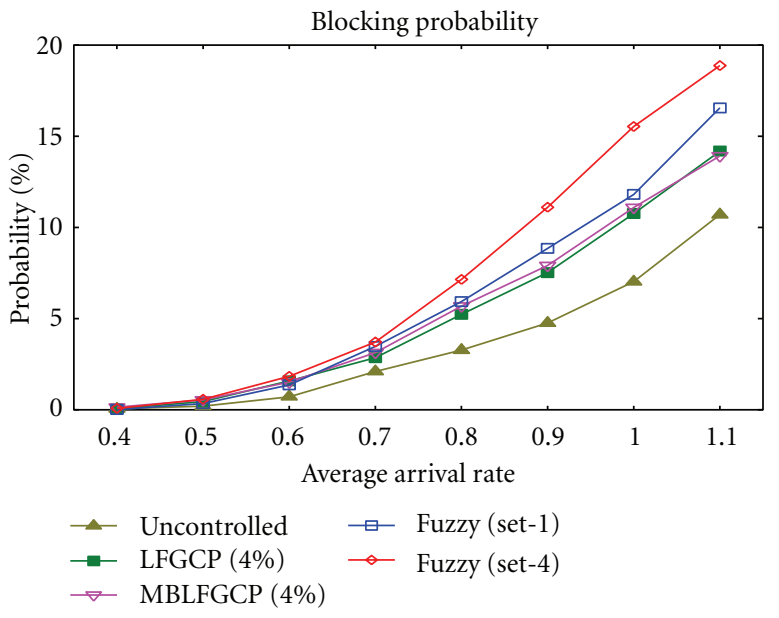

FIGURE 11: The comparison of blocking probability of all traffic types.

Mobile IPv6 networks. For simplicity, set- 1 and set- 4 are used as representatives of the four fuzzy sets. The proposed schemes have higher blocking probability and lower dropping probability. There is a tradeoff between increased $\mathrm{Pb}$ and decreased $\mathrm{Pd}$ according to the different values of the threshold parameters. For example, the set- 4 reserves

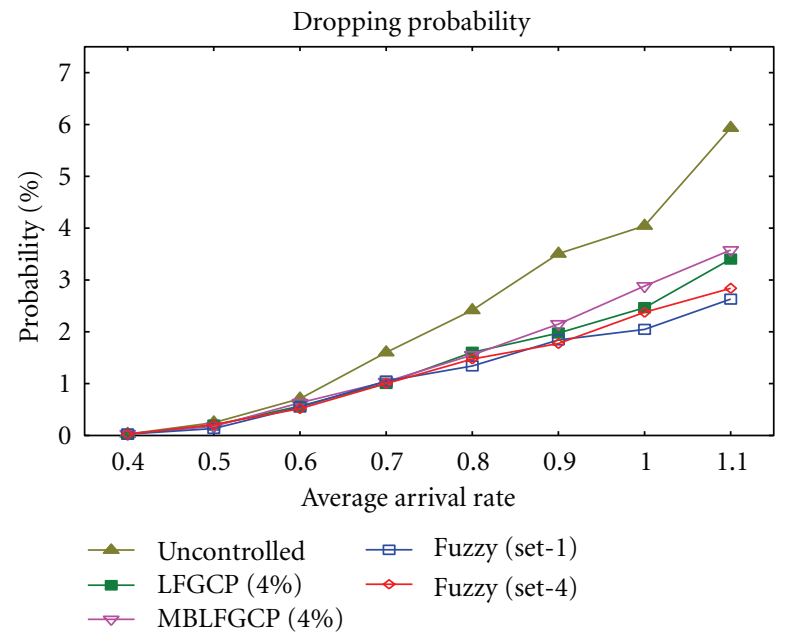

FIGURE 12: The comparison of dropping probability of all traffic types.

more bandwidth to the higher-priority traffic; the reserved bandwidth increased more than other schemes when the arrival rate increases. Owing to the same reason, the set4 provides the highest blocking probability and the lowest dropping probability. 


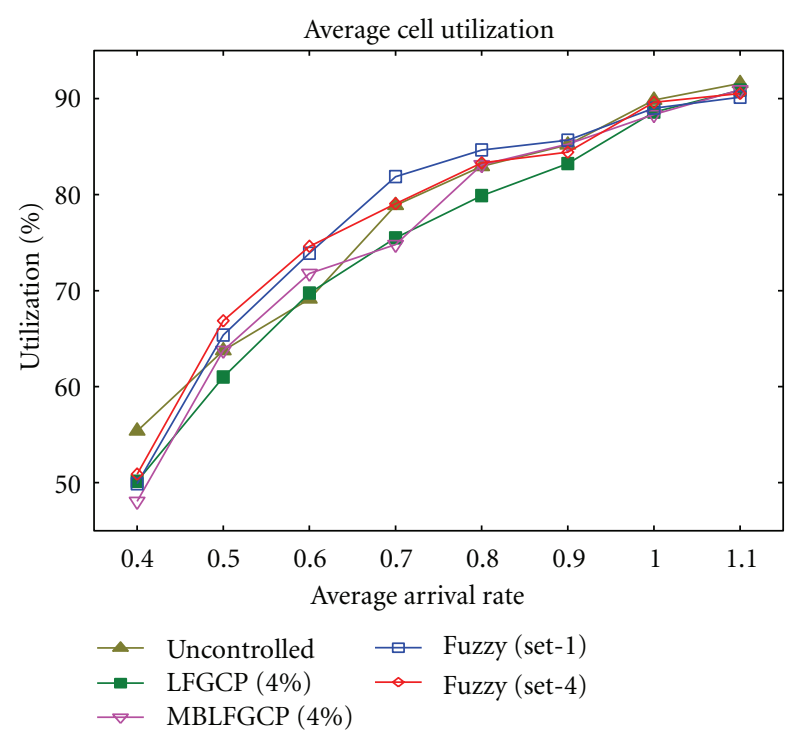

FIGURE 13: The average cell utilization.

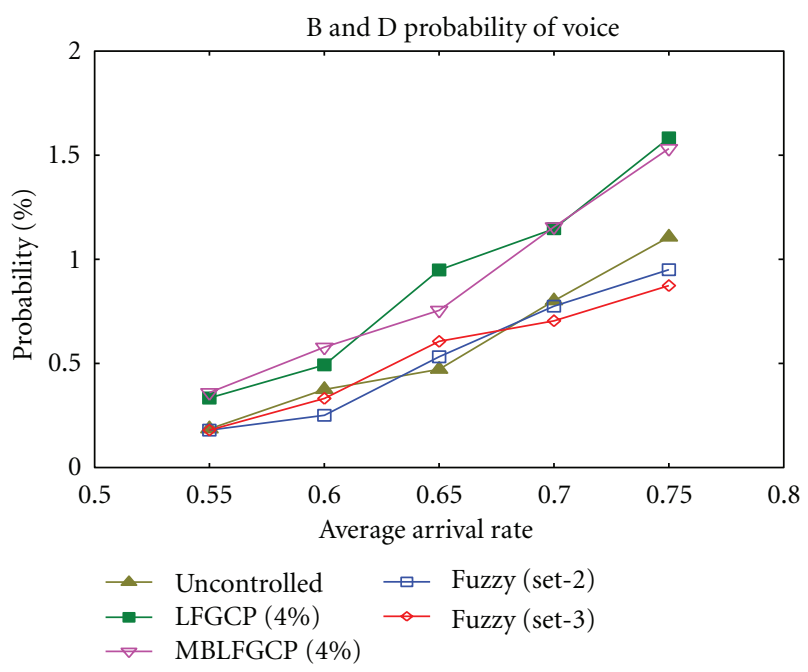

FIgURE 14: The blocking and dropping probability of voice traffic.

The average cell utilization, the ratio of used capacity with the total capacity in a cell, among no CAC (uncontrolled), LFGCP, MBLFGCP, and the proposed FLS-based CAC algorithm (set-1, set-4) are shown in Figure 13. Because the proposed scheme makes dynamical fuzzy decision of the threshold values, it is obvious that the proposed algorithm provides higher cell utilization than the others. The comparison of the blocking and dropping probability of voice traffic is shown in Figure 14, where the proposed algorithm provides much lower Pb\&d than MBLFGCP and LFGCP and also has a little better performance than the "uncontrolled" scheme. Because the video traffic needs much more bandwidth than other traffic, the video traffic is easier to be blocked or dropped. From the comparison of the blocking and dropping probability of video traffic that is shown in Figure 15, it is obvious that the proposed scheme has the best performance among all these CAC schemes.

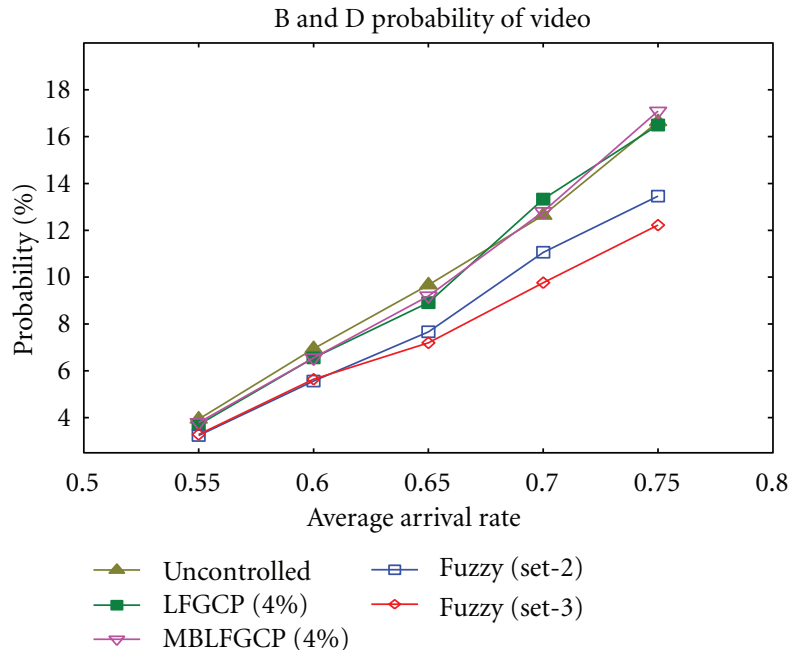

FIGURE 15: The blocking and dropping probability of video traffic.

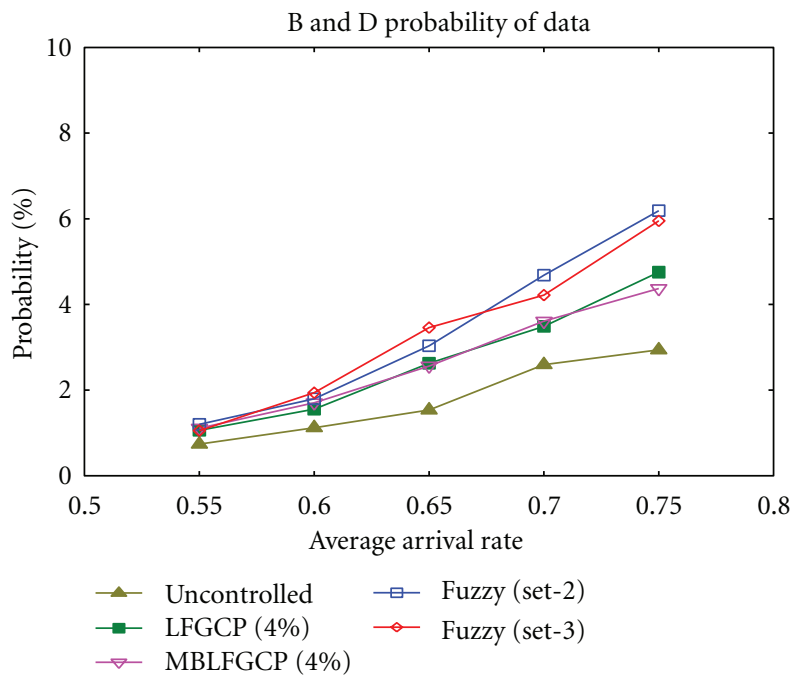

FIGURE 16: The blocking and dropping probability of data traffic.

The comparison of the blocking and dropping probability of data traffic is shown in Figure 16. Because the purpose of the proposed scheme tries to accept the real-time traffic at first, the proposed scheme has the worst performance for data traffic. The proposed scheme makes good performance for voice and video traffic with a sacrifice of the performance of data traffic.

\section{Conclusions}

We extend HMIPv6 Binding Update message to support CAC schemes. These CAC schemes can reduce the probability of the handoff dropping and cell overload and limit the new call blocking probability. We also propose a CAC scheme which incorporates multiple traffic types and adjusts the admission threshold dynamically using FIS to achieve better usage of resources. According to the simulation results, the proposed scheme presents good performance of voice and 
video traffic at the expenses of poor performance on data traffic. In future work, we will try to use genetic algorithm or adaptive neuron-fuzzy inference system (ANFIS) to make more complex and effective admission control schemes in HMIPv6 networks.

\section{References}

[1] D. Johnson, C. Perkins, and J. Arkko, "Mobility Support in IPv6," IETF RFC3775, pp.1-165, 2004.

[2] H. Soliman, C. Castelluccia, K. El Malki, and L. Bellier, "Hierarchical Mobile IPv6 Mobility Management (HMIPv6)," IETF RFC 5380, pp. 1-25, October 2008.

[3] I. F. Akyildiz, J. Xie, and S. Mohanty, "A survey of mobility management in next-generation all-IP-based wireless systems," IEEE Wireless Communications, vol. 11, no. 4, pp. 16-28, 2004.

[4] A. K. M. M. Hossain and K. Kanchanasut, "A handover management scheme for mobile IPv6 networks," in Proceedings of the 14th International Conference on Computer Communications and Networks (ICCCN '05), pp. 43-48, San Diego, Calif, USA, October 2005.

[5] D. Bruneo, L. Paladina, M. Paone, A. Puliafito, C. Papardo, and S. Sperone, "Call admission control in hierarchical mobile networks," in Proceedings of the 10th IEEE Symposium on Computers and Communications (ISCC '05), pp. 780-785, La Manga del Mar Menor, Cartagena, Spain, 2005.

[6] M. Ahmed, "Call admission control in wireless networks: a comprehensive survey," IEEE Communications Surveys \& Tutorials, vol. 7, pp. 49-68, 2005.

[7] G. le Grand and E. Horlait, "A predictive end-to-end QoS scheme in a mobile environment," in Proceedings of the 6th IEEE Symposium on Computers Communications (ISCC '01), pp. 534-539, Hammamet, Tunisia, July 2001.

[8] J. M. Moon, M. Y. Yun, G. S. Park, K. I. Kim, Y. J. Kim, and S. H. Kim, "QoS provisioning in domain based mobile IP networks," in Proceedings of the 57th IEEE Semiannual Vehicular Technology Conference, pp. 447-451, Jeju , Republic of Korea, April 2003.

[9] K. I. Kim, S. H. Kim, J. M. Moon, and Y. J. Kim, "Hierarchical admission control scheme for supporting mobility in mobile IP," in Proceedings of the IEEE Military Communications Conference (MILCOM '02), pp. 431-435, Anaheim, Calif, USA, October 2002.

[10] J. Hou and Y. Fang, "Mobility-based call admission control schemes for wireless mobile networks," Wireless Communications and Mobile Computing, vol. 1, no. 3, pp. 269-282, 2001.

[11] D. Hong and S. S. Rappaport, "Traffic model and performance analysis for cellular mobile radio telephone systems with prioritized and nonprioritized handoff procedures," IEEE Transactions on Vehicular Technology, vol. 35, no. 3, pp. 77-92, 1986.

[12] R. Ramjee, R. Nagarajan, and D. Towsley, "On optimal call admission control in cellular networks," in Proceedings of the IEEE Conference on Computer Communications (INFOCOM '96), pp. 43-50, San Francisco, Calif, USA, 1996.

[13] S. F. Yang and J. S. Wu, "Mobility based call admission control in hierarchical mobile IPv6 networks," in Proceedings of the 4th IASTED Asian Conference on Communication Systems and Networks (AsiaCSN '07), pp. 271-276, Phuket, Thailand, 2007.

[14] J. S. Wu, W. Y. Lin, and S. F. Yang, "Hierarchical mobile IPv6 mobility management in integrated Wi-Fi and WiMAX networks," Journal of Internet Technology, vol. 8, no. 3, pp. 253260, 2007.

[15] T. C. Chau, K. Y. M. Wong, and B. Li, "Optimal call admission control with QoS guarantee in a voice/data integrated cellular network," IEEE Transactions on Wireless Communications, vol. 5, no. 5, pp. 1133-1141, 2006.

[16] B. J. Hwang, I. S. Hwang, and L. F. Ku, "Adaptive bandwidth management and reservation scheme in heterogeneous wireless networks," Journal of Internet Technology, vol. 10, no. 3, pp. 237-244, 2009.

[17] S. Rito, P. Carvalho, and V. Freitas, "Admission control in multiservice IP networks: architectural issues and trends," IEEE Communications Magazine, vol. 45, no. 4, pp. 114-121, 2007.

[18] B. J. Hwang, I. S. Hwang, and S. C. Chang, "Adaptive resource management with fuzzy bandwidth control for multi-services in two-tier wireless networks," Journal of the Chinese Institute of Engineers, vol. 33, no. 2, 2010.

[19] J. M. Mendel, "Fuzzy logic systems for engineering: a tutorial," Proceedings of the IEEE, vol. 83, no. 3, pp. 345-377, 1995.

[20] W. Pedrycz, Fuzzy Control and Fuzzy Systems, John Wiley \& Sons, New York, NY, USA, 1993. 

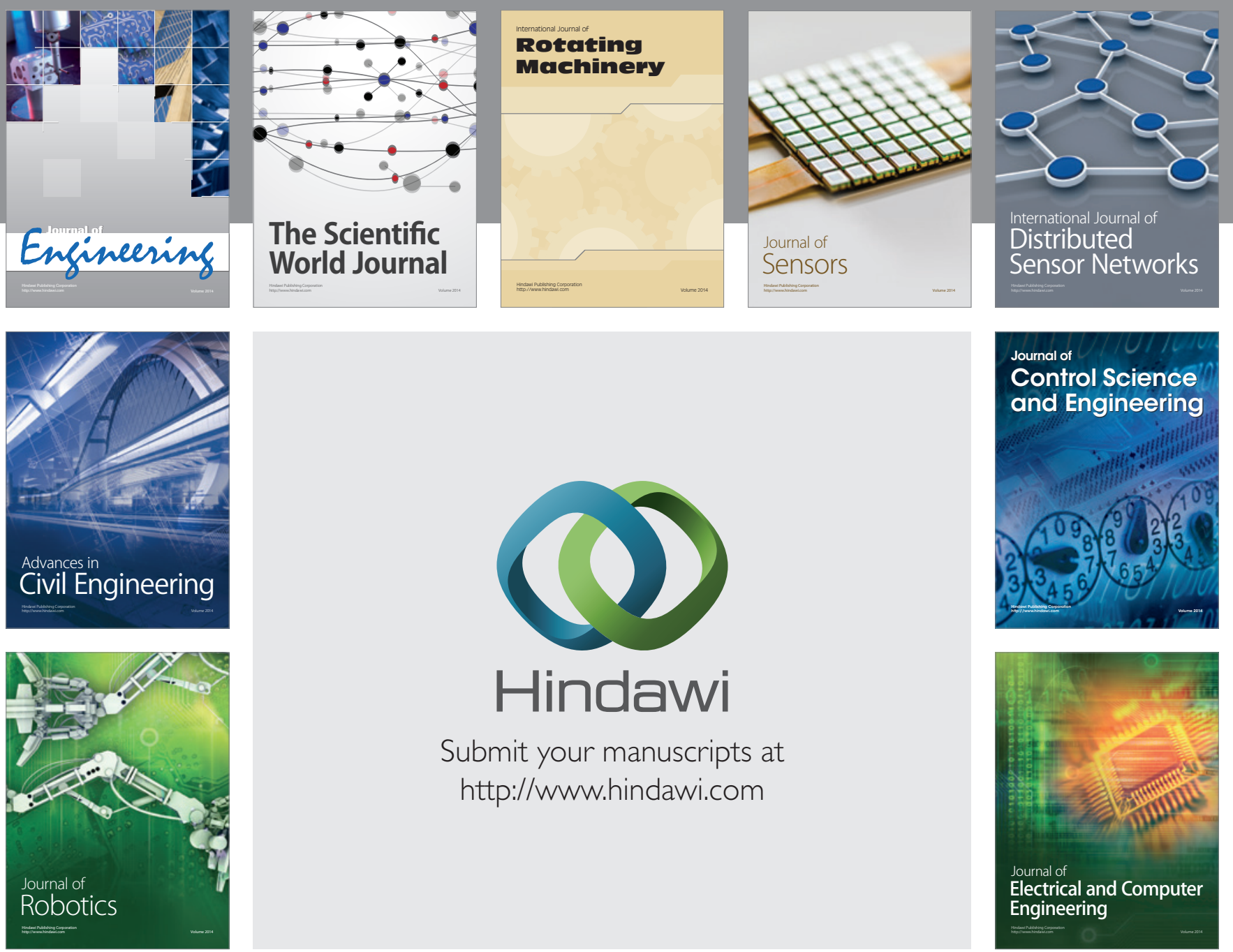

Submit your manuscripts at

http://www.hindawi.com
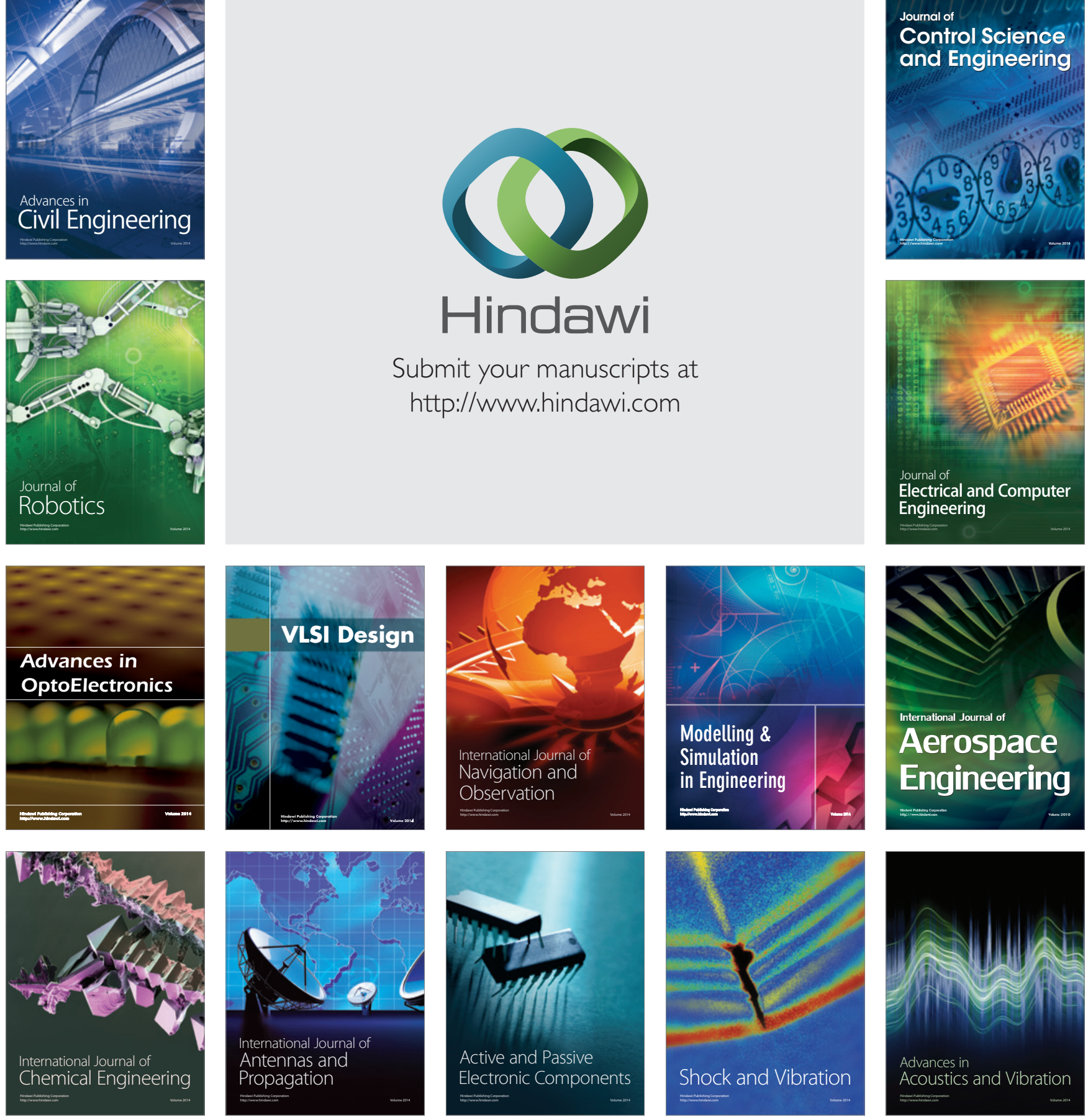